\title{
ON HYPERBOLIC 3-MANIFOLDS WITH SYMMETRIC HEEGAARD SPLITTINGS
}

\author{
Soo Hwan Kim And YangkoK Kim
}

\begin{abstract}
We construct a family of hyperbolic 3-manifolds by pairwise identifications of faces in the boundary of certain polyhedral 3-balls and prove that all these manifolds are cyclic branched coverings of the 3 sphere over certain family of links with two components. These extend some results from [5] and [10] concerning with the branched coverings of the whitehead link.
\end{abstract}

\section{Introduction}

There are two well known results about the realization of closed 3-manifolds. One is that any closed orientable 3-manifold can be obtained by Dehn surgeries on the components of an oriented link in the 3 -sphere. The other one says that any closed 3-manifold can be represented as a branched covering of some link in the 3-sphere. So if we consider a link in the 3-sphere, we can construct many classes of closed orientable 3-manifolds by considering its branched coverings or Dehn surgeries along it. The description of closed 3-manifolds as polyhedral 3-balls, whose finitely many boundary faces are glued together in pairs, is a further standard way to construct 3-manifolds (see [3], [4], [10], [11], and [12]). If the polyhedral 3-ball admits a geometric structure and the face identification is performed by means of geometric isometries, then the same geometric structure is inherited by the quotient manifold (see [10], [12], and [15]). Many authors have studied the connections between the face identification procedure and the representation of closed 3-manifolds as branched coverings of the 3sphere. In [10] Helling, Kim and Mennicke considered a family of polyhedral 3 -balls $\mathcal{P}_{n}$ depending on a positive integer $n$, and for any coprime positive integers $n$ and $k$, they defined a pairwise gluing of faces in the boundary of $\mathcal{P}_{n}$ yielding a closed orientable 3 -manifold $\mathcal{M}_{n, k}$. In the sequel, they proved that $\mathcal{M}_{n, k}$ is an $n$-fold strongly cyclic covering of the 3 -sphere branched over the Whitehead link and classified, up to isometry, those coverings. More general

Received August 16, 2004; Revised March 22, 2008.

2000 Mathematics Subject Classification. Primary 57M12, 57M25; Secondary 57M50, $57 \mathrm{~N} 10$.

Key words and phrases. crystallization, cyclic branched covering, symmetric Heegaard splitting. 
cases (that is the branched coverings of the Whitehead link which are not necessarily strongly cyclic) were handled by A. Cavicchioli and L. Paoluzzi [5], when $\operatorname{gcd}(n, k)=d>1$.

In this paper we consider the following classes of links $\mathcal{W}_{m}$ and $\mathcal{L}_{(m, d)}$ for positive integers $m$ and $d$ as shown in Figure 1 , where the index $m-1$ in Figure 1a denotes the number of half twists and each $L_{i}$ in box denotes the $\left(\frac{1}{m}\right)$-rational tangle. We note that $\mathcal{W}_{m}$ is a link of two components which is the rational $\left(\frac{4 m}{2 m-1}\right)$-tangle. Moreover $\mathcal{W}_{m}$ and $\mathcal{L}_{(m, d)}$ extend the Whitehead link $\mathcal{W}_{2}$ and $\mathcal{L}_{(2,1)}$. We construct an infinite family of 3 -manifolds $\mathcal{M}(2 m+1, n, k)$ by the identification of oppositely oriented boundary faces of a polyhedral 3cell $\mathcal{P}(2 m+1, n)$ for positive integers $m, n, k$. Then we shall deal with the combinatorial representation of $\mathcal{M}(2 m+1, n, k)$ by a special class of edgecolored graphs, called crystallizations (see for example [2], [8], [6], and [14]).

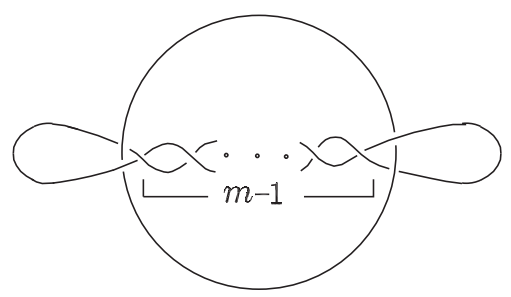

a. The $\operatorname{link} \mathcal{W}_{m}$

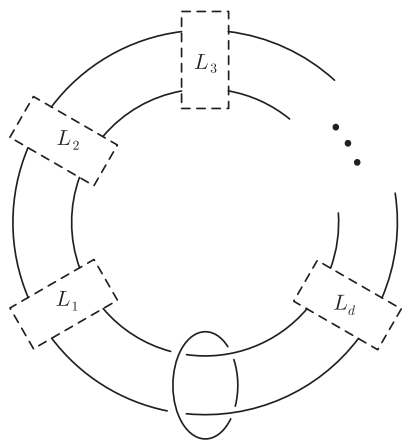

b. $\mathcal{L}_{(m, d)}$

Figure 1

By applying $L C G$ moves on crystallizations ([13] and [16]), we prove that $\mathcal{M}(2 m+1,2 d, d)$ is a 2 -fold strongly cyclic covering of the 3 -sphere branched over $\mathcal{L}_{(m, d)}$. In the sense of Birman and Hilden [1], we obtain the result that the symmetric extension of a Heegaard splitting for $\mathcal{M}(2 m+1,2 d, d)$ represents $\mathcal{M}(2 m+1, n, k)$ as $(n / d)$-fold strongly cyclic coverings of the 3 -sphere branched over $\mathcal{L}_{(m, d)}$, where $\operatorname{gcd}(n, k)=d$. Moreover $\mathcal{M}(2 m+1, n, k)$ is an $n$-fold cyclic covering of the 3 -sphere branched over $\mathcal{W}_{m}$, where the branched indices of its components are $n$ and $n / d$, respectively. We note that $\mathcal{L}_{(m, d)}$ is hyperbolic for $m>1$ and so does $\mathcal{M}(2 m+1, n, k)$ for $m>1$. The results extend the corresponding ones of Helling-Kim-Mennicke [10] and Cavicchioli-Paoluzzi [5], where $m=2$ and $\operatorname{gcd}(n, k)=1$, and $m=2, \operatorname{gcd}(n, k)=d>2$, respectively.

\section{Construction of the manifold $\mathcal{M}(2 m+1, n, k)$}

In this section we construct an infinite family of 3-manifold $\mathcal{M}(2 m+1, n, k)$ by considering a combinatorial polyhedron together with an identification of 


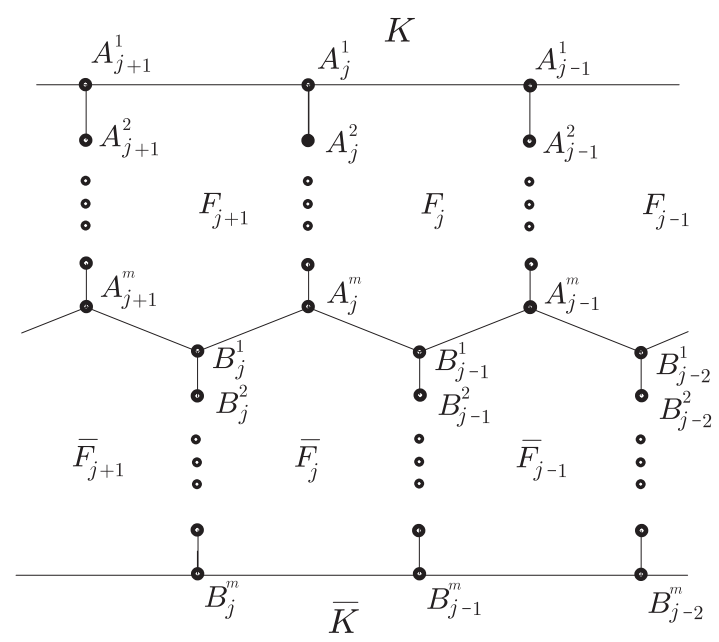

FigURE 2. $\mathcal{P}(2 m+1, n)$

pairs of faces on its boundary. For positive integers $m, n$ and $k$, let $\mathcal{P}(2 m+$ $1, n)$ be a polyhedron whose boundary, which can be regarded as the 2 -sphere, consists of two $n$-gons in the northern and southern hemispheres, and $2 n(2 m+$ $1)$-gons in the equatorial zone as shown in Figure 2. Then $\mathcal{P}(2 m+1, n)$ has $2 n+2$ faces, $2 n m+2 n$ edges and $2 n m$ vertices.

We define the boundary cycles of two $n$-gons and $2 n(2 m+1)$-gons as follow:

$$
\begin{aligned}
& K \quad: \quad A_{1}^{1} A_{2}^{1} \cdots A_{n}^{1} \\
& \bar{K} \quad: \quad B_{1}^{m} B_{2}^{m} \cdots B_{n}^{m} \\
& F_{j} \quad: \quad A_{j}^{m} A_{j}^{m-1} \cdots A_{j}^{1} A_{j-1}^{1} A_{j-1}^{2} \cdots A_{j-1}^{m} B_{j-1}^{1} \\
& \bar{F}_{j} \quad: \quad A_{j}^{m} B_{j}^{1} B_{j}^{2} \cdots B_{j}^{m} B_{j-1}^{m} B_{j-1}^{m-1} \cdots B_{j-1}^{2} B_{j-1}^{1}
\end{aligned}
$$

for $j=1, \ldots, n$ (see Figure 2).

We now define the face identification of $2 n(2 m+1)$-gons as follows: for each $j=1, \ldots, n$,

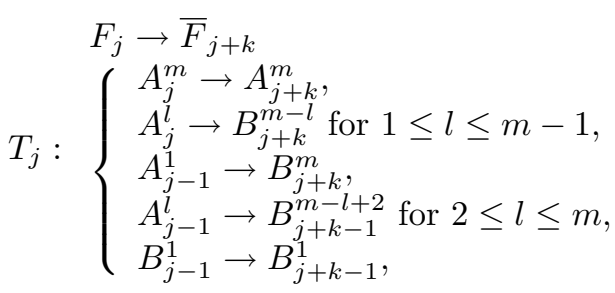

where the indices are taken $\bmod n$.

Consider the oriented edges:

$$
x_{j}=\left(A_{j}^{1}, A_{j+1}^{1}\right) \text { and } u_{j}=\left(B_{j-1}^{1}, A_{j}^{m}\right) .
$$




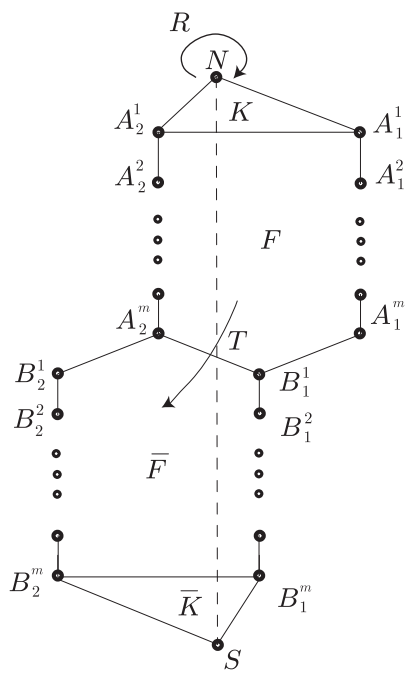

Figure 3. The quotient space $\mathcal{P}(2 m+1, n) / \sim$

Then the identifications $T_{j}$ naturally induce the face identification of two $n$ gons. Moreover each oriented edge $u_{j}$ has $\frac{n}{d}$ equivalent edges for $j=1,2, \ldots, n$. Now we calculate the Euler characteristic of the cellular complex $\mathcal{K}(2 m+1, n, k)$ induced by the face identifications of the polyhedron $\mathcal{P}(2 m+1, n)$. We note that there is a rotational symmetry $\sim$ by

$$
A_{j}^{i} \rightarrow A_{j-1}^{i} \text { and } B_{j}^{i} \rightarrow B_{j-1}^{i} \text { for all } i=1,2, \ldots, m \text { and } j=1,2, \ldots, n
$$

of $\mathcal{P}(2 m+1, n)$. Thus it suffices to consider our case in the quotient space $\mathcal{P}(2 m+1, n) / \sim$ with $\operatorname{gcd}(n, k)=1$ described in Figure 3, where $N$ and $S$ are the centers of two $n$-gons in $\mathcal{P}(2 m+1, n)$.

Indeed, it is easy to see that all edges of two $(2 m+1)$-gons except $\left(B_{1}^{1}, A_{2}^{m}\right)$ are equivalent under the repeated applications of composition actions of $Q, R$ and $T$;

$$
Q:\left\{\begin{array}{l}
A_{2}^{1} \rightarrow B_{2}^{m} \\
A_{1}^{1} \rightarrow B_{1}^{m} \\
N \rightarrow S
\end{array} \quad \text { and } R:\left\{\begin{array}{l}
N \rightarrow N \\
S \rightarrow S \\
A_{2}^{i} \rightarrow A_{1}^{i} \\
B_{2}^{i} \rightarrow B_{1}^{i}
\end{array}\right.\right.
$$

for all $i=1,2, \ldots, m$, and

$$
T:\left\{\begin{array}{l}
A_{1}^{1} \rightarrow B_{2}^{m} \\
A_{1}^{l} \rightarrow B_{1}^{m-l+2} \quad \text { for } 2 \leq l \leq m \\
B_{1}^{1} \rightarrow B_{1}^{1} \\
A_{2}^{m} \rightarrow A_{2}^{m} \\
A_{2}^{l} \rightarrow B_{2}^{m-l} \quad \text { for } 1 \leq l \leq m-1 .
\end{array}\right.
$$


This means that the complex $\mathcal{K}(2 m+1, n, k)$ has $d$ vertices, $n+d$ edges, $n+1$ two-cells and 1 three-cell. So it follows that the resulting complex $\mathcal{M}(2 m+$ $1, n, k)$ is a closed oriented 3-manifold by the theorem of Seifert and Threlfall: $a$ complex formed by identifying the faces of a polyhedron will be a closed manifold if and only if its Euler characteristic equals zero.

\section{Crystallizations and LCG moves}

We introduce crystallizations and $L C G$ moves (for detail, see [13] and [16]). For a given multigraph $\Gamma, V(\Gamma)$ and $E(\Gamma)$ denote the sets of vertices and edges of $\Gamma$ (both finite) respectively. An edge-coloration on a graph $\Gamma=(V(\Gamma), E(\Gamma))$ is a map $\gamma: E(\Gamma) \rightarrow \Delta=\{0,1,2,3\}$ such that $\gamma(e) \neq \gamma(f)$ for any two adjacent edges $e, f$. A pair $(\Gamma, \gamma)$ is called a 4 -colored graph if $\Gamma$ is regular of valency 4 . For a subset $\Delta^{\prime}$ of $\Delta$, we set $\Gamma_{\Delta^{\prime}}=\left(V(\Gamma), \gamma^{-1}\left(\Delta^{\prime}\right)\right)$. Each connected component of $\Gamma_{\Delta^{\prime}}$ is called a $\Delta^{\prime}$-residue of degree $k$, where $k$ is the order of the component. The number of $\{i j\}$-residues of $(\Gamma, \gamma)$ is denoted by $g_{i j}(\Gamma, \gamma)$. A 4-colored graph $(\Gamma, \gamma)$ is said to be contracted if $\Gamma_{\Delta \backslash\{i\}}$ is connected for each $i \in \Delta$. If $(\Gamma, \gamma)$ is a 4-colored graph, then the associated pseudocomplex $K(\Gamma)$ is defined as follows:

(1) take a 3-simplex $\sigma^{3}(v)$ for each $v \in V(\Gamma)$ and label its vertices by different elements of $\Delta$;

(2) if $v, w \in V(\Gamma)$ are joined by an $i$-colored edge, then identify the 2-faces of $\sigma^{3}(v)$ and $\sigma^{3}(w)$ opposite to the vertices labelled by $i$, so that equally labelled vertices are identified together.

In this case $(\Gamma, \gamma)$ is said to represent $K(\Gamma)$ and every space homeomorphic to it. A contracted 4-colored graph representing a closed connected 3-manifold $M$ is said to be a crystallization of $M$.

For the drawing of a crystallization we first fix two arbitrary colors, for example, 0 and 1 . Then we draw all $\{01\}$-residues as circles, and draw all the third colored edges, say 2 -colored edges, by connecting $\{01\}$-residues. Finally we express each 3 -colored edge by denoting the initial and terminal vertices by $a, a^{*}$ or simply $a, a$ if there is no confusion. This expression is called a crystallization based on $(01 ; 2)$. In $(\Gamma, \gamma)$ an $\{i j\}$-residue $\{a, b, c, d\}$ of degree 4 is called standard if four vertices $a, b, c$ and $d$ in $\Gamma$ have the following two properties:

(1) $a, b, c$ and $d$ are vertices of a $\{i j\}$-residue of degree 4;

(2) $a, b, c$ and $d$ are vertices of mutually distinct $\Delta \backslash\{i j\}$-residues.

In particular, when $\{a, b, c, d\}=\left\{a, a^{*}, b, b^{*}\right\}$, we simply write a standard $\{i j\}$-residue $\{a, b\}$ or a standard 2-residue $\{a, b\}$ for a standard $\{i j\}$-residue $\left\{a, a^{*}, b, b^{*}\right\}$ of degree 4 . We note that every closed connected 3 -manifold admits a crystallization, and that a manifold can have non-isomorphic crystallizations. However there is a set of moves, called moves I, II, and A, which connects any two crystallizations of a manifold (see [7]). We now introduce a $L C G$ move which is equivalent to moves I, II, and A. 


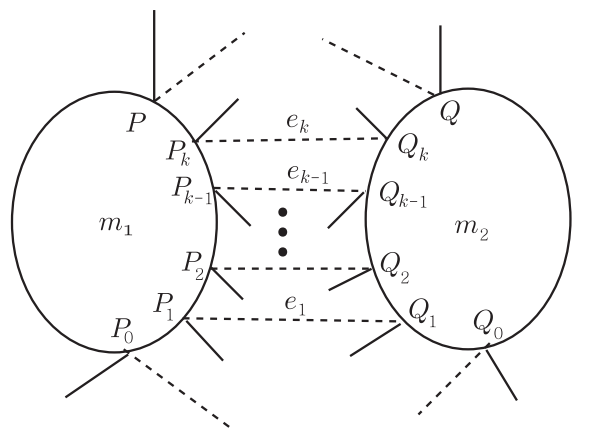

Figure 4. An extended 1-dipole

Let $m_{1}$ and $m_{2}$ be two distinct $\{01\}$-residues in $\Gamma$ with $\Delta=\{0,1,2,3\}$. We say that $m_{1}$ and $m_{2}$ are joined by consecutive 3 -colored edges $e_{1}, e_{2}, \ldots, e_{k}$ if there exists a partial ordering of vertices $P_{0}, P_{1}, \ldots, P_{k-1}, P_{k}, P$ of $m_{1}$ and $Q_{0}, Q_{1}, \ldots, Q_{k-1}, Q_{k}, Q$ of $m_{2}$ such that

(1) $P_{i}$ is joined to $Q_{i}$ by a 3 -colored edge $e_{i}$ for $1 \leq i \leq k$;

(2) $\overline{P_{0} P_{1}}$ and $\overline{Q_{0} Q_{1}}$ belong to a $\{03\}$ or $\{13\}$-residue of degree greater than 4

(3) $\overline{P_{k} P}$ and $\overline{Q_{k} Q}$ belong to a $\{03\}$ or $\{13\}$-residue of degree greater than 4.

Let $m_{1}$ and $m_{2}$ be two distinct $\{01\}$-residues in $\Gamma$ which are joined by consecutive 3 -colored edges $e_{1}, e_{2}, \ldots, e_{k}$. Then a partial subgraph of $\Gamma$ formed by vertices $P_{1}, \ldots, P_{k}$ of $m_{1}$ and $Q_{1}, \ldots, Q_{k}$ of $m_{2}$, joined by consecutive 3 -colored edges is said to be an extended 1-dipole if $m_{1}$ and $m_{2}$ belong to different $\{012\}$ residues (see Figure 4). In this case, we simply say that $\Gamma$ has an extended 1-dipole generated by $\left(P_{1}, \ldots, P_{k} ; Q_{1}, \ldots, Q_{k}\right)$. We recall that a subgraph of $\Gamma$ formed by two vertices $X, Y$ joined by 2 edges with colors $i, j$ will be called a dipole $\{X, Y\}$ of type 2 if and only if $X$ and $Y$ belong to distinct components of $\Gamma_{\Delta \backslash\{i, j\}}$.

Let $\Gamma$ have an extended 1-dipole consisting of two $\{01\}$-residues $m_{1}, m_{2}$ and consecutive 3 -edges $e_{1}, e_{2}, \ldots, e_{k}$. Then we construct a 4 -colored graph $\Gamma^{\prime}$ from $\Gamma$ as follows:

(1) Remove all 0,1 and 3 -colored edges ending at $P_{i}$ or $Q_{i}$, and all $P_{i}$ or $Q_{i}$ for $1 \leq i \leq k$ from $\Gamma$.

(2) Connect 2-colored edges ending at $Q_{i}$ and $P_{i}$ for $1 \leq i \leq k$.

(3) Connect 0 or 1-colored edges ending at $Q$ and $P$, and $Q_{0}$ and $P_{0}$

In this case we say that $\Gamma^{\prime}$ is obtained from $\Gamma$ by eliminating an extended 1-dipole or $\Gamma$ is obtained from $\Gamma^{\prime}$ by adding an extended 1-dipole.

We say that $\Gamma^{\prime}$ is obtained from $\Gamma$ by a linear cut-and-glue move (or a $L C G$ move) if there exists a non-contracted 4-colored graph $\bar{\Gamma}$ such that $\bar{\Gamma}$ is obtained 
from $\Gamma$ by adding an extended 1-dipole and $\Gamma^{\prime}$ is obtained from $\bar{\Gamma}$ by eliminating an extended 1-dipole. Two crystallizations are said to be $L C G$-equivalent if they can be joined by a finite sequence of $L C G$ moves.

Theorem 1 ([7] and [13]). Let $M$ and $M^{\prime}$ be closed 3-manifolds, and $(\Gamma, \gamma)$ and $\left(\Gamma^{\prime}, \gamma^{\prime}\right)$ be two crystallizations of $M$ and $M^{\prime}$ respectively. Then the following statements are equivalent:

(1) $M$ is homeomorphic with $M^{\prime}$,

(2) $(\Gamma, \gamma)$ and $\left(\Gamma^{\prime}, \gamma^{\prime}\right)$ are $(I, I I)$-equivalent,

(3) $(\Gamma, \gamma)$ and $\left(\Gamma^{\prime}, \gamma^{\prime}\right)$ are A-equivalent,

(4) $(\Gamma, \gamma)$ and $\left(\Gamma^{\prime}, \gamma^{\prime}\right)$ are LCG-equivalent.

Theorem 2. Let $M$ be a closed 3-manifold and $(\Gamma, \gamma)$ be a crystallization of M. If $(\Gamma, \gamma)$ has a standard $\{23\}$-residue, then there is a crystallization $\left(\Gamma^{\prime}, \gamma^{\prime}\right)$ of $M$ such that $g_{01}\left(\Gamma^{\prime}, \gamma^{\prime}\right)=g_{01}(\Gamma, \gamma)-1$.

Proof. Let $(\Gamma, \gamma)$ have a standard $\{23\}$-residue $\{a, b\}$. Then we assume, without loss of generality, that $(\Gamma, \gamma)$ contains a part shown in Figure $5 \mathrm{a}$, where the circles denote $\{01\}$-residues and the dashed lines denote 2 -colored edges.

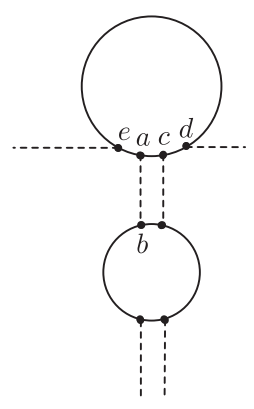

a. $(\Gamma, \gamma)$

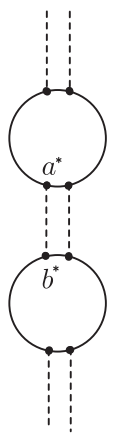

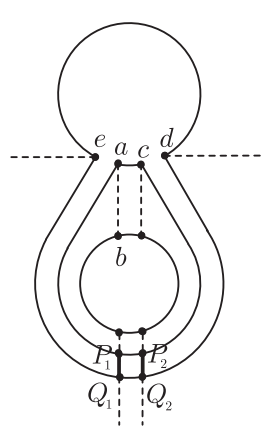

b. $(\bar{\Gamma}, \bar{\gamma})$

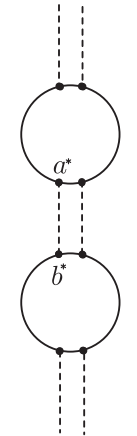

$$
\bar{\gamma})
$$




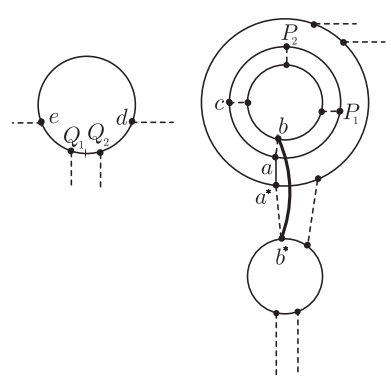

a. $(\bar{\Gamma}, \bar{\gamma})$

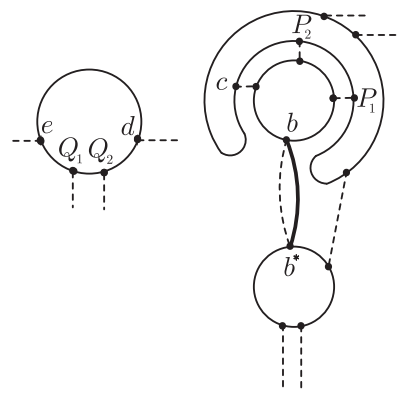

b.

Figure 6

We note that $(\bar{\Gamma}, \bar{\gamma})$ has a standard $\{23\}$-residue $\{a, b\}$. Moreover $(a ; a)$ generates an extended 1-dipole. By eliminating it, we have a 4-colored graph with a dipole $\left\{b, b^{*}\right\}$ of type 2 (see Figure $6 \mathrm{~b}$ ). Indeed a crystallization in Figure $6 \mathrm{~b}$ is obtained from $(\Gamma, \gamma)$ by a $L C G$-move. Finally, by cancelling the dipole $\left\{b, b^{*}\right\}$ of type 2 , we have a crystallization $\left(\Gamma^{\prime}, \gamma^{\prime}\right)$ of $M$ such that $g_{01}\left(\Gamma^{\prime}, \gamma^{\prime}\right)=g_{01}(\Gamma, \gamma)-1$.

Let $(\Gamma, \gamma)$ be a crystallization of a closed 3-manifold $M$ with a standard 2-residue $\{a, b\}$. Then a crystallization $\left(\Gamma^{\prime}, \gamma^{\prime}\right)$ of $M$ constructed by using the method in the proof of Theorem 2 is said to be generated from $(\Gamma, \gamma)$ by $L C G$ moves.

\section{The manifold $\mathcal{M}(2 m+1, n, k)$ as a branched covering}

There are two presentations corresponding to a spine of $\mathcal{M}(2 m+1, n, k)$. One is the description of polyhedral 3-balls, whose finitely many boundary faces are glued together in pairs, and the other one is a $(n / d)$-symmetric Heegaard splitting (or $(n / d)$-symmetric crystallization). The latter is easily obtained from a combinatorial complex triangulating $\mathcal{M}(2 m+1, n, k)$ by pairwise identification of boundary 2-cells. By [1], $\mathcal{M}(2 m+1, n, k)$ is an $(n / d)$-fold cyclic covering of the 3 -sphere branched over a link of bridge number $\leq \frac{p-1+g}{p-1}$, where $g$ is the genus of $\mathcal{M}(2 m+1, n, k)$ and $p=\frac{n}{d}$. By the rotational symmetry, it suffices to prove the result in case $n=2 d$. We now consider the polyhedral schemata $\mathcal{P}(2 m+1,2 d)$, which defines the closed orientable 3 -manifold $\mathcal{M}(2 m+1,2 d, d)$ as a quotient of a triangulated 3 -ball $B^{3}$ by pairwise identification of its boundary 2 -cells (see Figure 2 ). Triangulate $\mathcal{P}(2 m+1,2 d)$ into a simplicial complex $K(2 m+1,2 d)$ by using stellar subdivisions (for example see a triangulation of $K(7,4)$ in Figure 7$)$. We note that outside of the exterior circle we have a vertex and the corresponding stellar subdivision. The configuration is a simplicial tessellation of the 2 -sphere $\partial B^{3}$ consisting of $2(2 m+1) d+2$ vertices, $6(2 m+1) d$ edges, and $4(2 m+1) d$ triangles. Let $w$ be a point in the interior 


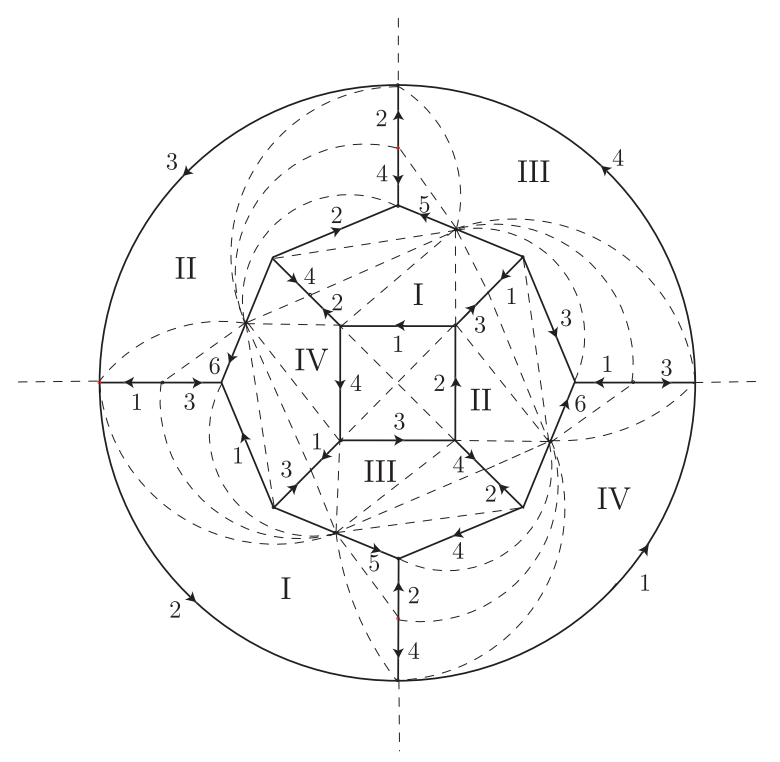

Figure 7. $K(7,4)$

of $B^{3}$. Now $K(2 m+1,2 d)$ is just the simplicial join from $w$ on the above tessellation. Identify the two copies of each triangle in $\partial K(2 m+1,2 d)$ so that the corresponding oriented edges carrying the same label are glued together. The identification produces a pseudocomplex $\widetilde{K}(2 m+1,2 d, d)$ which triangulates $\mathcal{M}(2 m+1,2 d, d)$ consisting of $2 d+2$ vertices, $2 d+2+4(2 m+1) d$ edges, $8(2 m+1) d$ triangles, and $4(2 m+1) d$ tetrahedra. Indeed $\widetilde{K}(2 m+1,2 d, d)$ is a colored complex. We now construct the 4 -colored graph $\widetilde{\Gamma}(2 m+1,2 d, d)$ associated to $\widetilde{K}(2 m+1,2 d, d)$ as follows. The vertices of $\widetilde{\Gamma}(2 m+1,2 d, d)$ are the elements of

$$
\widetilde{V}(2 m+1,2 d, d)=\left(\{0,2 d+1\} \times \mathbb{Z}_{2 d}\right) \cup\left(\{1,2, \ldots, 2 d\} \times \mathbb{Z}_{4 m}\right) .
$$

For coloring edges of $\widetilde{\Gamma}(2 m+1,2 d, d)$ we consider the following four fixed-pointfree involutions on $\widetilde{V}(2 m+1,2 d, d)$ :

$$
\begin{aligned}
& v_{0}(i, j)=\left(i, j+(-1)^{j}\right), \\
& v_{1}(i, j)=\left(i, j-(-1)^{j}\right), \\
& v_{2}(i, j)= \begin{cases}(2 d+1, i-1) & \text { if } j=0, \\
(0,1-i) & \text { if } j=2 m, \\
\left(i+(-1)^{i+1} \mu(j), j\right) & \text { otherwise, }\end{cases} \\
& v_{3}(i, j)= \begin{cases}(i+d, 2 m+1-j) & \text { if } i \in\{1,2, \ldots, d\}, i \text { odd }, \\
(i+d, 2 m-1-j) & \text { if } i \in\{1,2, \ldots, d\}, i \text { even }, \\
(2 d+1,1-j) & \text { if } i=0,\end{cases}
\end{aligned}
$$


where $\mu: \mathbb{Z}_{4 m} \backslash\{0,2 m\} \rightarrow\{+1,-1\}$ is the function defined by

$$
\mu(j)= \begin{cases}+1 & \text { if } 1 \leq j \leq 2 m-1 \\ -1 & \text { if } 2 m+1 \leq j \leq 4 m-1\end{cases}
$$

and the arithmetic is either $\bmod 2 d$ or $\bmod 4 \mathrm{~m}$. We now define the 4-colored graph $\widetilde{\Gamma}(2 m+1,2 d, d)$ as follows: for each $i \in \Delta$ two vertices $x$ and $y$ in $\widetilde{V}(2 m+1,2 d, d)$ are joined by an $i$-colored edge if and only if $y=v_{i}(x)$. The geometrical shape of $\widetilde{\Gamma}(2 m+1,2 d, d)$ consists of $2 d$ circles $C_{i}$ which are $\{01\}$ residues of length $4 m$ cyclically set on the plane following the natural order of the set $\{1,2, \ldots, 2 d\}$, and of two circles $C_{0}, C_{2 d+1}$ of length $2 d$ which are $\{01\}$ residues of length $2 d$. For each $i \in\{0, \ldots, 2 d\}$, the vertex set of $C_{i}$ consists of pairs $(i, j)$ for any $j \in \mathbb{Z}_{4 m}$. The vertex sets of $C_{0}$ and $C_{2 d+1}$ consist of pairs $(i, j)$ for any $j \in \mathbb{Z}_{2 d}$. We cyclically order the vertices $(i, j)$ of each $C_{i}$ following the natural order of $j$ in $\mathbb{Z}_{4 m}$ (or $\mathbb{Z}_{2 d}$ ) so that all these orderings induce the clockwise (resp. anti-clockwise) orientation of the plane when $i$ is odd (resp. even). There are exactly $2 m-12$-colored edges between $C_{i}$ and $C_{i+1}$ (resp. $C_{i}$ and $C_{i-1}$ ) for any $i=1, \ldots, 2 d$ (here $0 \equiv 2 d$ ). There is exactly one 2 colored edge between $C_{i}$ and $C_{2 d+1}$ (resp. $C_{i}$ and $C_{0}$ ) for any $i=1, \ldots, 2 d$. Furthermore there are exactly $4 m$ 3-colored edges between $C_{i}$ and $C_{i+d}$ for any $i=1, \ldots, d$, and $2 d 3$-colored edges between $C_{0}$ and $C_{2 d+1}$. For the simple notation of vertices, we order vertices linearly by the lexicographic ordering:

$$
(i, j)<(k, l) \text { if } i<k \text {, or } i=k \text { and } j<l .
$$

First we do numbering for the vertices of $C_{1}$ to $C_{d}$ by the lexicographic ordering. That is, we use numbers 1 to $4 m d$ for the numbering of the vertices of $C_{1}$ to $C_{d}$, for example 1 for $(1,0), 2$ for $(1,1)$ and so on. We then order the vertices of $C_{0}$ by using numbers $4 m d+1$ to $4 m d+2 d$. For the rest of vertices in $C_{d+1}, \ldots, C_{2 d+1}$ we do as follows; if the vertex is connected to a vertex numbered $k$ by a 3 -colored edge, then we number the vertex by $k$. (For example see Figure 8 for the crystallization of $\widetilde{\Gamma}(2 m+1,4,2)$ with numbered vertices.) Summarizing the above argument we have:

Lemma 1. The closed orientable 3 -manifold $\mathcal{M}(2 m+1,2 d, d)$ is represented by a 4-colored graph $\widetilde{\Gamma}(2 m+1,2 d, d)$.

We also note that in the above method of stellar subdivision which triangulates $\mathcal{P}(2 m+1,2 d)$ into a simplicial complex $K(2 m+1,2 d)$ is independent of $d$. Thus we can prove the following theorems by using the special numbers $d$ without loss of generality. Indeed we use the same method as one in [5].

Theorem 3. The closed orientable 3-manifold $\mathcal{M}(2 m+1,2 d, d)$ is a 2-fold strongly cyclic covering of the 3 -sphere branched over $\mathcal{L}_{(m, d)}$, where $d$ is even. Furthermore, $\mathcal{M}(2 m+1,2 d, d)$ is a $2 d$-fold cyclic branched covering of $\mathcal{W}_{m}$ in the 3-sphere, where the branched indices of its components are $2 d$ and 2 , respectively. 


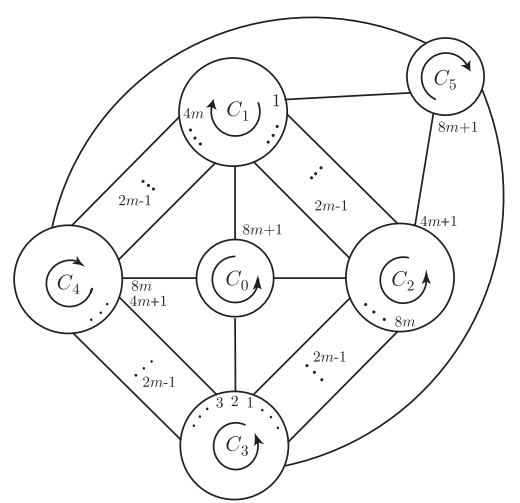

Figure 8. The 4-colored graph $\widetilde{\Gamma}(2 m+1,4,2)$

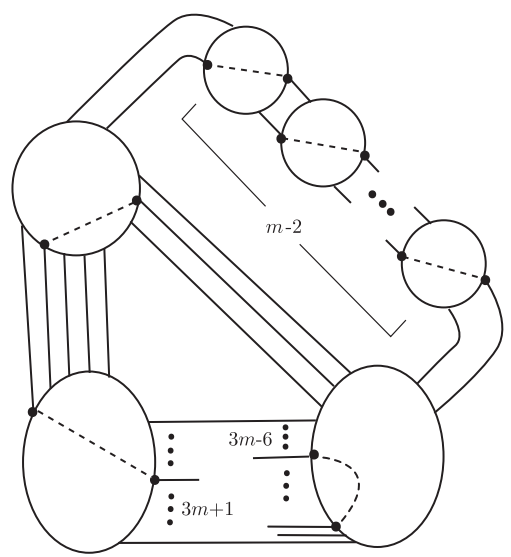

FiguRE 9. A 2-symmetric crystallization $\Gamma(2 m+1,4,2)$ inducing an link

Proof. We note that $\mathcal{M}(2 m+1,2 d, d)$ can be represented by a 4-colored graph $\widetilde{\Gamma}(2 m+1,2 d, d)$ by Lemma 1 . Thus it suffices to prove that a 2 -symmetric crystallization $\Gamma(2 m+1,2 d, d)$ is obtained from $\widetilde{\Gamma}(2 m+1,2 d, d)$ by a finite sequence of $L C G$-moves. For simplicity we now restrict our case to $d=2$. One can immediately extend the construction for the general cases by a simple iteration. We let a subgraph $\widetilde{\Gamma}(2 m+1,4,2)$ be regularly embedded in the plane. To see standard 2-residues specifically, we consider a crystallization based on $(02 ; 1)$. Then it consists of $(4 m-4)\{02\}$-colored cycles of length 4 and four $\{02\}$-colored cycles of length 6 . Now the crystallization $\Gamma(2 m+$ $1,4,2)$ contains $(4 m+2)$ standard $\{02\}$-residues. By applying a $L C G$ move for a standard $\{02\}$-residue, we have a 4 -colored graph $\Gamma^{\prime}(2 m+1,4,2)$ such 
that $g_{02}\left(\Gamma^{\prime}(2 m+1,4,2)\right)=g_{02}(\Gamma(2 m+1,4,2))-1$. Note that $g_{02}(\Gamma(2 m+$ $1,4,2))=4 m$ and $g_{02}\left(\left(\Gamma^{\prime}(2 m+1,4,2)\right)\right)=4 m-1$. Repeating this procedure for $(3 m-1)$ standard $\{02\}$-residues, we obtain a crystallization $\bar{\Gamma}(2 m+1,4,2)$ with $g_{02}(\bar{\Gamma}(2 m+1,4,2))=4 m-(3 m-1)=m+1$, which yields a 2 -symmetric crystallization as shown in Figure 9, where dashed lines denote the axis of a 2symmetric Heegaard splitting induced by a 2-symmetric crystallization. Thus $\mathcal{M}(2 m+1,4,2)$ is the 2 -fold covering of the 3 -sphere branched over a link. Using Reidemeister moves, it is immediate to verify that this link is equivalent to a 3 -bridge link $\mathcal{L}_{(m, 2)}$.

For the second statement, we simply note that $\mathcal{L}_{(m, d)}$ has a component which can be regarded as the axis of symmetry of order $d$. This symmetry produces a $d$-fold cyclic branched covering of $\mathcal{W}_{m}$ in the 3 -sphere, where the branched indices of its components are $2 d$ and 2 , respectively.

We now explicitly describe the procedures described in Theorem 3 for $\mathcal{M}(7$, $4,2)$ which is the case $m=3$ and $d=2$. On the basis of the triangulation in Figure 7 we have a crystallization $\widetilde{\Gamma}(7,4,2)$ based on $(01 ; 2)$ as shown in Figure 10a. We now change the crystallization based on $(01 ; 2)$ to one based on $(12 ; 0)$ to see standard $\{03\}$-residues (see Figure $10 \mathrm{~b})$. We note that there

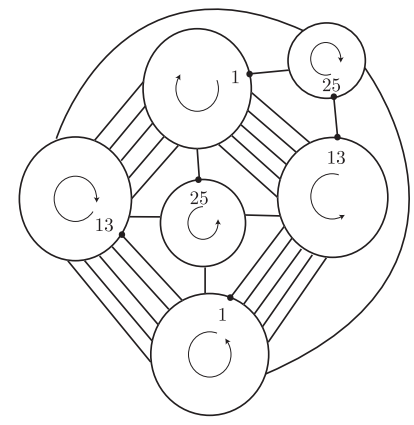

a. Based on $(01 ; 2)$

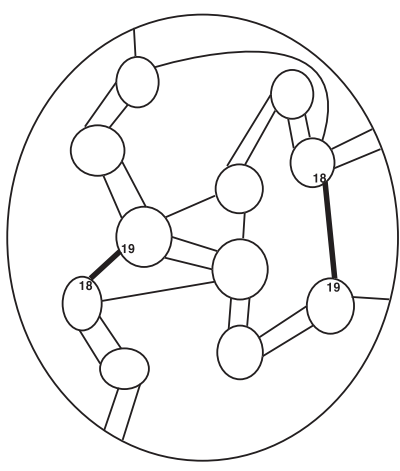

b. Based on $(12 ; 0)$

FiguRE 10

is a standard $\{03\}$-residue $\{18,19\}$. Applying a $L C G$ move for $\{18,19\}$, we have a 4-colored graph as shown in Figure 11a. We can do the same job for a standard $\{03\}$-residue $\{13,24\}$ in a 4 -colored graph. One application of a $L C G$ move reduces the number of $\{03\}$-residues by one. We can continue this procedure 8 times to get a crystallization shown in Figure 11b which is a 2symmetric crystallization of $\mathcal{M}(7,4,2)$. Thus $\mathcal{M}(7,4,2)$ is the 2 -fold covering of the 3-sphere branched over a 4-bridge link. Using Reidemeister moves, it is immediate to verify that this link is equivalent to $\mathcal{L}_{(3,2)}$. 


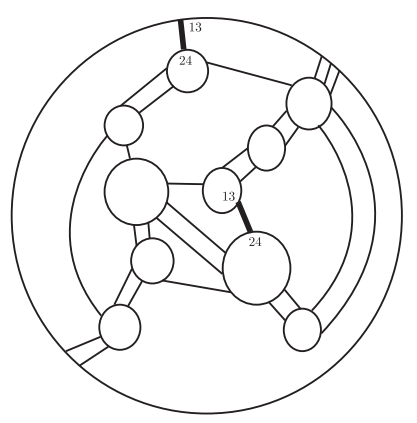

a. Based on $(01 ; 2)$

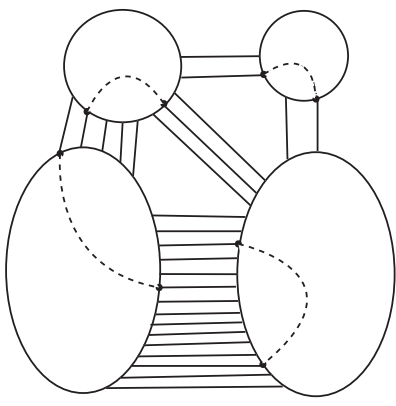

b. 2-symmetric crystallization

FIGURe 11

Theorem 4. The closed orientable 3-manifold $\mathcal{M}(2 m+1,2 d, d)$ is a 2-fold strongly cyclic covering of the 3-sphere branched over $\mathcal{L}_{(m, d)}$, where $d$ is odd. Furthermore, $\mathcal{M}(2 m+1,2 d, d)$ is $2 d$-fold cyclic branched covering of $\mathcal{W}_{m}$ in the 3-sphere, where the branched indices of its components are $2 d$ and 2 , respectively.

Proof. We consider the case $d=3$ that can be immediately extended to the construction for the general cases by a simple iteration as in Theorem 3 . That is, we claim that $\mathcal{P}(2 m+1,6)$ can be represented by a 2 -symmetric crystallization $\Gamma(2 m+1,6,3)$. Let $\mathcal{P}(2 m+1,6)$ be the polyhedral schemata which defines the closed orientable 3 -manifold $\mathcal{M}(2 m+1,6,3)$ as a quotient of a triangulated 3 -ball $B^{3}$ by pairwise identification of its boundary 2-cells. Triangulate $\mathcal{P}(2 m+1,6)$ into a simplicial complex $\widetilde{K}(2 m+1,6,3)$. There are two distinct ways to triangulate $\mathcal{P}(2 m+1,6)$ depending on the parity of $m$. For example, we see two triangulations for $\mathcal{P}(7,6)$ and $\mathcal{P}(9,6)$, where $m=3$ and 4 , respectively in Figure 12. We can handle the general cases by a natural extension.

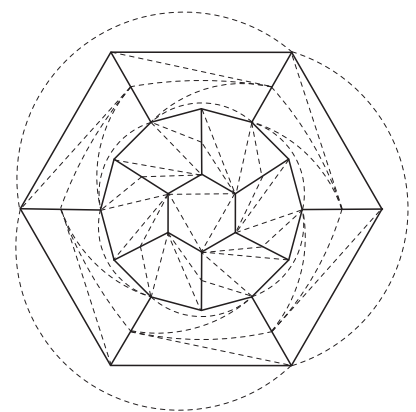

a. $\widetilde{K}(7,6,3)$

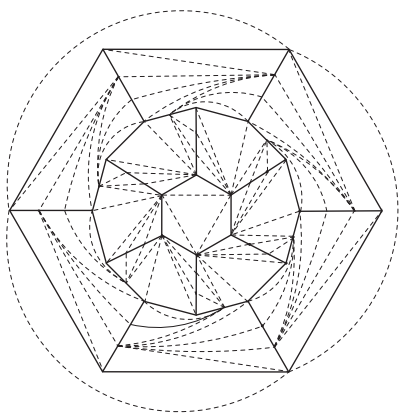

b. $\widetilde{K}(9,6,3)$

FiguRE 12 
By the same way in the proof of Theorem 3, we can construct a crystallization $\widetilde{\Gamma}(2 m+1,6,3)$ associated to $\widetilde{K}(2 m+1,6,3)$ as follows.

Case i) $m=2 t+1$ and $t \geq 0$.

The vertices of $\widetilde{\Gamma}(2 m+1,6,3)$ are the elements of

$$
\widetilde{V}(2 m+1,6,3)=\{(i, j(i)) \mid i=1,2, \ldots, 18\} \cup\left\{O, O^{\prime}\right\},
$$

where

$$
\left\{\begin{array}{cl}
1 \leq j(i) \leq m+3 & \text { if } i=1,2,3 \\
1 \leq j(i) \leq 2 m+2 & \text { if } i=4,5,6 \\
1 \leq j(i) \leq m+3 & \text { if } i=7,8,9 \\
1 \leq j(i) \leq 2 & \text { if } i=10,11,12 \\
1 \leq j(i) \leq 2 m-4 & \text { if } i=13,14,15 \\
1 \leq j(i) \leq 2 m-8 & \text { if } i=16,17,18
\end{array}\right.
$$

For colored edges, we consider a permutation $\eta$ which is of order 3 :

$$
\eta=(1,2,3)(4,5,6)(7,8,9)(10,11,12)(13,14,15)(16,17,18)
$$

on $X=\{0,1,2, \ldots, 18\}$ and a subset $V_{1}$ of $\widetilde{V}(2 m+1,6,3)$;

$$
V_{1}=\{(i, j(i)) \mid i=1,4,7,10,13,16\} \cup\left\{O, O^{\prime}\right\}
$$

with the following edge-colorations;

$$
\begin{aligned}
& v_{0}\left(O^{\prime}\right)=O \text {, } \\
& v_{0}(1,1)=(11,2), v_{0}(1,2)=(12,1), v_{0}(1,3)=(9, m+2) \text {, } \\
& v_{0}(1,4)=(9, m+3), v_{0}(1, j)=(18, m+1-j), 5 \leq j \leq m \text {, } \\
& v_{0}(1, m+1)=(6,4), v_{0}(1, m+2)=(6,5) \text {, } \\
& v_{0}(4,1)=(1,2), v_{0}(4,2)=(5, m+4), v_{0}(4,3)=(5, m+5) \text {, } \\
& v_{0}(4,4)=(2, m+1), v_{0}(4,5)=(2, m+2) \text {, } \\
& v_{0}(4, j)=(16,2 m+2), 6 \leq j \leq m+3, v_{0}(4, m+4)=(6,2) \text {, } \\
& v_{0}(4, m+5)=(6,3), v_{0}(4, j)=(18,3 m-2-j), m+6 \leq j \leq 2 m+1 \text {, } \\
& v_{0}(4,2 m+2)=(9,1) \text {, } \\
& v_{0}(7,1)=(5,2 m+2), v_{0}(7,2)=(5,1), v_{0}(7, j)=(14, m-1-j), 3 \leq j \leq m \text {, } \\
& v_{0}(7, m+1)=(1, m+3), v_{0}(7, m+2)=(2,3), v_{0}(7, m+3)=(2,4) \text {, } \\
& v_{0}(10,2)=(3,1), v_{0}(10,1)=(2,2) \text {, } \\
& v_{0}(14, j)= \begin{cases}(9, m+1-j), & \text { if } 1 \leq j \leq m-2, \\
(5,2 m+2-j), & \text { if } m-1 \leq j \leq 2 m-4,\end{cases} \\
& v_{0}(16, j)= \begin{cases}(2, m+1-j), & \text { if } 1 \leq j \leq m-4, \\
(5,3 m-2-j), & \text { if } m-3 \leq j \leq 2 m-8,\end{cases} \\
& v_{1}(1, j)=\left(1, j-(-1)^{j}\right) \text {, where } j \in \mathbb{Z}_{m+3} \text {, } \\
& v_{1}(4, j)=\left(4, j+(-1)^{j}\right) \text {, where } j \in \mathbb{Z}_{2 m+2} \text {, } \\
& v_{1}(13, j)=(13, j+1), 1<j<2 m-2, j \text { even, } \\
& v_{1}(13,1)=(3, m+3) \text {, and } v_{1}(13,2 m-4)=(3, m+2) \text {, } \\
& v_{1}(16,2 j)=(16,2 j-1), 1<2 j \leq 2 m-8, j \text { even, } j \neq m-3 \text {, } \\
& v_{1}(10,1)=(7, m+2) \text {, and } v_{1}(10,2)=O^{\prime} \text {, }
\end{aligned}
$$




$$
\begin{aligned}
& v_{2}(1,1)=O, \\
& v_{2}(4,1)=(13, m-2) \text { and } v_{0}(4,2)=(13, m-1), \\
& v_{2}(4,3)=(16,2 m-8), \text { and } v_{0}(4,4)=(17,1), \\
& v_{2}(4, j)=(8, m+4-j), 5 \leq j \leq m+2, \\
& v_{2}(4, j)=(1,2 m+4-j), m+3 \leq j \leq 2 m+2, \\
& v_{2}(7, j)=\left(7, j-(-1)^{j}\right), \text { where } j \in \mathbb{Z}_{m+3}, \\
& v_{2}(10,1)=(8, m), \text { and } v_{2}(10,2)=(8, m+1), \\
& v_{2}(13, j)=(13, j-1), 1<2 j<2 m-4, j \text { even, } j \neq m-1, \\
& v_{2}(16, j)=(16, j+1), 1<j<2 m-8, j \text { even, } \\
& v_{3}(1, j)=\left(1, j+(-1)^{j}\right), \text { where } j \in \mathbb{Z}_{m+3}, \\
& v_{3}(4, j)=\left(4, j-(-1)^{j}\right), \text { where } j \in \mathbb{Z}_{2 m+2}, \\
& v_{3}(7, j)=\left(7, j-(-1)^{j}\right), \text { where } j \in \mathbb{Z}_{m+3}, \\
& v_{3}(10,1)=(10,2), \\
& v_{3}(13, j)=(13,2 m-3-i), \\
& v_{3}(16, j)=(16,2 m-7-i) .
\end{aligned}
$$

We define an action of $\eta$ on $V$ by

$$
\begin{aligned}
\eta(v, w)= & (\eta(v), w) \text { for }(v, w) \in V \backslash\left\{O, O^{\prime}\right\}, \text { and } \\
& \eta \text { fixes } O \text { and } O^{\prime} .
\end{aligned}
$$

Furthermore for edge-colorations,

$$
\text { if } y=v_{i}(x) \text { for } x, y \text { in } V \text {, then we define } \eta(y)=v_{\eta(i)}(\eta(x)) .
$$

Since $V_{1} \cup \eta\left(V_{1}\right) \cup \eta^{2}\left(V_{1}\right)=V$, a function $\eta$ and an edge-coloration of $V_{1}$ produce a crystallization $\widetilde{\Gamma}(2 m+1,6,3)$ associated to $\widetilde{K}(2 m+1,6,3)$.

For example, we consider the case $\widetilde{\Gamma}(7,6,3)$. One can easily extend to the general case $\widetilde{\Gamma}(2 m+1,6,3)$ by a simple extension. We have an crystallization based on $(01 ; 2)$ associated to $\widetilde{K}(7,6,3)$ in Figure $13 \mathrm{a}$. There exist 10 standard $\{01\}$-residues. By the same method in Theorem 3 and example following, we reduce the number of $\{01\}$-residues.

Note that the result does not depend on the choice of a standard 2-residue. First we remove a standard 2-residue $\{20,28\}$ as shown in Figure 13a. We apply a $L C G$ move for $\{20,28\}$ to get Figure $13 \mathrm{~b}$ which has a standard 2residue $\{9,10\}$. We apply a $L C G$ move for $\{9,10\}$. We can continue the same procedure until we remove all standard 2-residues. Note that each time we can reduce the genus by one. Finally we obtain the 2-symmetric crystallization $\Gamma(7,6,3)$ as shown in Figure 14a, where dashed lines denote the axis of a 2 symmetric Heegaard splitting induced by a 2 -symmetric crystallization. 


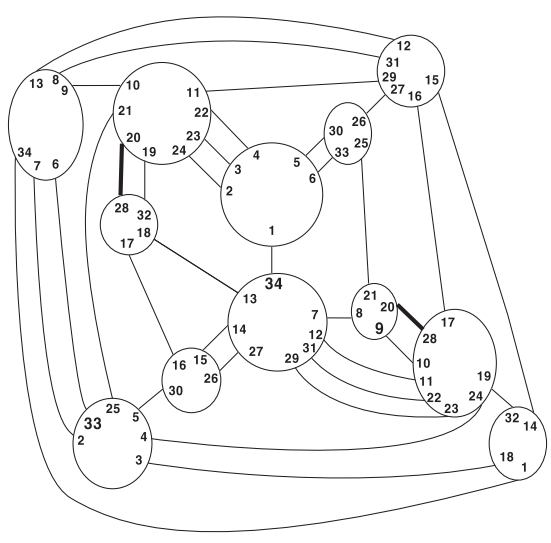

a.

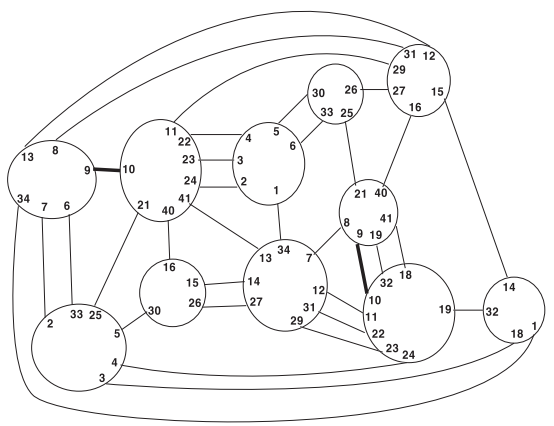

b.

Figure 13

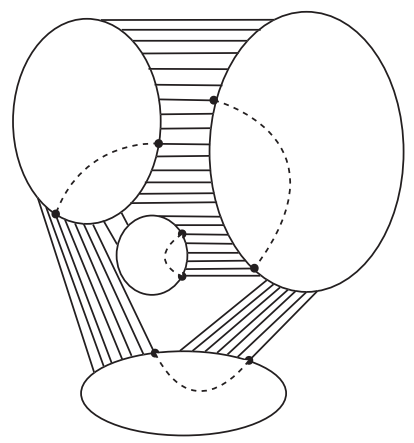

a. $\Gamma(7,6,3)$

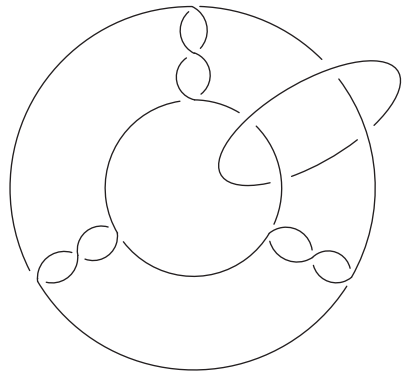

b. The link $\mathcal{L}_{(3,3)}$

FiguRE 14

By applying Reidemeister moves on a 4-bridge link induced by a 2 -symmetric crystallization $\Gamma(7,6,3)$, we can easily verify that the link is equivalent to the link $\mathcal{L}_{(3,3)}$ as shown in Figure $14 \mathrm{~b}$.

Case ii) $m=2 t$

We can apply the same argument for case i) with the following edge-colorations of $V_{1}$;

$v_{0}(O)=O^{\prime}$

$v_{0}(1,1)=(11,2), v_{0}(1,2)=(12,1), v_{0}(1, j)=(18, m+2-j), 3 \leq j \leq m+1$,

$v_{0}(1, m+2)=(6,2)$,

$v_{0}(1, m+3)=(6,3)$,

$v_{0}(1, m+4)=(7, m+2)$,

$v_{0}(4,1)=(9,2), v_{0}(4,2)=(2, m+2)$, 


$$
\begin{aligned}
& v_{0}(4, j)= \begin{cases}(15,2 m+2-j), & \text { if } 3 \leq j \leq m+2, \\
(18,3 m-3-j), & \text { if } m+3 \leq j \leq 2 m-1,\end{cases} \\
& v_{0}(4,2 m)=(9,1) \text {, } \\
& v_{0}(7,1)=(5,2 m), v_{0}(7,2)=(5,1), v_{0}(7, j)=(18, m+2-j), 3 \leq j \leq m+1 \text {, } \\
& v_{0}(7, m+2)=(1, m+4), v_{0}(7, m+3)=(2,3), v_{0}(7, m+4)=(2,4), \\
& v_{0}(10,2)=(3,1), v_{0}(10,1)=(2,2) \text {, } \\
& v_{0}(13, j)=\left\{\begin{array}{cl}
(9, m+2-j), & \text { if } 1 \leq j \leq m-1, \\
(5,2 m-2-j), & \text { if } m \leq j \leq 2 m-2,
\end{array}\right. \\
& v_{0}(16, j)= \begin{cases}(2, m+2-j), & \text { if } 1 \leq j \leq m-3, \\
(5,3 m-3-j), & \text { if } m-2 \leq j \leq 2 m-6,\end{cases} \\
& v_{1}(1, j)=\left(1, j+(-1)^{j}\right) \text {, where } j \in \mathbb{Z}_{m+3}, \\
& v_{1}(4, j)=\left(4, j-(-1)^{j}\right) \text {, where } j \in \mathbb{Z}_{2 m+2} \text {, } \\
& v_{1}(7, j)=\left(7, j+(-1)^{j}\right) \text {, } \\
& v_{1}(13, j)=(13,2 m-3-j), 1 \leq j \leq 2 m-4 \text {, } \\
& v_{1}(16, j)=(16,2 m-5-j), 1 \leq j \leq 2 m-6 \text {, } \\
& v_{1}(10,1)=(10,2) \text {, } \\
& v_{2}(1,1)=O \text {, } \\
& v_{2}(1, j)=(4,2 m+2-j), 2 \leq j \leq m+1, \\
& v_{2}(1, m+2)=(8,2) \text {, } \\
& v_{2}(1, m+3)=(14,2 m-2), v_{2}(1, m+4)=(14,1) \text {, } \\
& v_{2}(4,1)=(13, m-1), v_{2}(4,2)=(16,1), \\
& v_{2}(4, j)=(8, m+3-j), 3 \leq j \leq m, \\
& v_{2}(7, j)=\left(7, j-(-1)^{j}\right) \text {, where } j \in \mathbb{Z}_{m+4} \text {, } \\
& v_{2}(10,1)=(8, m+1), v_{2}(10,2)=(8, m+2) \text {, } \\
& v_{2}(13, j)=(13, j-1), 1<j<2 m-2, j \text { even, } j \neq m \text {, } \\
& v_{2}(16, j)=(16, j+1), 1<j<2 m-6 \text { and } j \text { even, } \\
& v_{2}(16,2 m-6)=(13, m) \text {, }
\end{aligned}
$$

and

$$
\begin{aligned}
v_{3}(1, j) & =\left(1, j-(-1)^{j}\right), \text { where } j \in \mathbb{Z}_{m+3}, \\
v_{3}(4, j) & =\left(4, j+(-1)^{j}\right), \text { where } j \in \mathbb{Z}_{2 m+2}, \\
v_{3}(7, m+3) & =(10,1), v_{3}(7, m+4)=(16, m-3), \\
v_{3}(7,1) & =(16, m-2), \\
v_{3}(10,2) & =O^{\prime}, \\
v_{3}(13,1) & =(3, m+4), v_{3}(13,2 m-4)=(3, m+3), \\
v_{3}(13, j) & =(13, j+1), j \text { even, } 1<j<2 m-4, \\
v_{3}(16, j) & =(16, j-1), j \text { even, } 1 \leq j \leq 2 m-6, j \neq m-2 .
\end{aligned}
$$


The same argument for Theorem 3 can be applied for the second statement.

We denote by $\mathcal{O}_{n / d}\left(\mathcal{L}_{(m, d)}\right)$ an orbifold whose underlying space is the 3 sphere and whose singular set is $\mathcal{L}_{(m, d)}$ with branched index $n / d$. Similarly by $\mathcal{O}_{n, n / d}\left(\mathcal{W}_{m}\right)$ we denote an orbifold whose underlying space is the 3 -sphere and whose singular set is $\mathcal{W}_{m}$ with the branched indices of its components are $n$ and $n / d$, respectively. Then we obtain the following result.

Theorem 5. The closed connected orientable 3 -manifold $\mathcal{M}(2 m+1, n, k)$ is an $(n / d)$-fold strongly cyclic covering of the 3 -sphere branched over $\mathcal{L}_{(m, d)}$, where $d=(n, k)$. Furthermore, $\mathcal{M}(2 m+1, n, k)$ is an $n$-fold cyclic branched covering of $\mathcal{W}_{m}$ in the 3 -sphere, where the branched indices of its components are $n$ and $n / d$, respectively.

Proof. We note that $\mathcal{M}(2 m+1,2 d, d)$ is a 2 -fold strongly cyclic branched covering of the 3 -sphere over $\mathcal{L}_{(m, d)}$ by Theorems 3 and 4 . Hence $\mathcal{M}(2 m+1,2 d, d)$ admits a 2 -symmetric Heegaard splitting. As a sense of [1] $\mathcal{M}(2 m+1, n, k)$ admits an $(n / d)$-symmetric Heegaard splitting by the rotational symmetry, where $d=(n, k)$. By [1], $\mathcal{M}(2 m+1, n, k)$ is an $(n / d)$-fold strongly cyclic branched covering of the 3 -sphere over $\mathcal{L}_{(m, d)}$. We note that $\mathcal{L}_{(m, d)}$ has an unknotted component which is the axis of $d$-symmetry of the chain (see Figure 1 ). Hence $\mathcal{O}_{n, n / d}\left(\mathcal{W}_{m}\right)$ is the 3 -sphere branched over $\mathcal{W}_{m}$ whose branched indices of its components are $n$ and $n / d$, respectively. That is, we have the following commutative diagram of branched coverings:

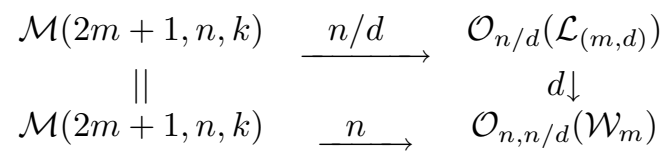

where the labels of the maps indicate the degree of the covering.

Acknowledgements. I would like to thank the referee for an improvement of the article and other helpful comments.

\section{References}

[1] J. S. Birman and H. M. Hilden, Heegaard splittings of branched coverings of $\mathbb{S}^{3}$, Trans. Amer. Math. Soc. 213 (1975), 315-352.

[2] J. Bracho and L. Montejano, The combinatorics of colored triangulations of manifolds, Geom. Dedicata 22 (1987), no. 3, 303-328.

[3] A. Cavicchioli, F. Hegenbarth, and A. C. Kim, A geometric study of Sieradski groups, Algebra Colloq. 5 (1998), no. 2, 203-217.

[4] , On cyclic branched coverings of torus knots, J. Geom. 64 (1999), no. 1-2, 55-66.

[5] A. Cavicchioli and L. Paoluzzi, On certain classes of hyperbolic 3-manifolds, Manuscripta Math. 101 (2000), no. 4, 457-494.

[6] M. Ferri, Crystallisations of 2-fold branched coverings of $\mathbb{S}^{3}$, Proc. Amer. Math. Soc. 73 (1979), no. 2, 271-276. 
[7] M. Ferri and C. Gagliardi, Crystallisation moves, Pacific J. Math. 100 (1982), no. 1, 85-103.

[8] M. Ferri, C. Gagliardi, and L. Grasselli, A graph-theoretical representation of PLmanifolds - a survey on crystallizations, Aequationes Math. 31 (1986), no. 2-3, 121-141.

[9] C. Gagliardi, A combinatorial characterization of 3-manifold crystallizations, Boll. Un. Mat. Ital. A (5) 16 (1979), no. 3, 441-449.

[10] H. Helling, A. C. Kim, and J. L. Mennicke, Some honey-combs in hyperbolic 3-space, Comm. Algebra 23 (1995), no. 14, 5169-5206.

[11] _ A geometric study of Fibonacci groups, J. Lie Theory 8 (1998), no. 1, 1-23.

[12] H. M. Hilden, M. T. Lozano, and J. M. Montesinos-Amilibia, The arithmeticity of the figure eight knot orbifolds, Topology '90 (Columbus, OH, 1990), 169-183, Ohio State Univ. Math. Res. Inst. Publ., 1, de Gruyter, Berlin, 1992.

[13] Y. H. Im and S. H. Kim, Heegaard splittings of the Brieskorn homology spheres that are equivalent after one stabilization, Note Mat. 20 (2001), no. 1, 53-63.

[14] S. L. Lins and A. Mandel, Graph-encoded 3-manifolds, Discrete Math. 57 (1985), no. 3, 261-284.

[15] L. Paoluzzi and B. Zimmermann, On a class of hyperbolic 3-manifolds and groups with one defining relation, Geom. Dedicata 60 (1996), no. 2, 113-123.

[16] H. J. Song, Y. H. Im, and K. H. Ko, LCG moves in crystallizations, Geom. Dedicata 76 (1999), no. 3, 229-251.

[17] W. P. Thurston, The Geometry and Topology of 3-Manifolds, Lect. Notes, Princeton, N.J., Princeton Univ. Press, 1980.

Soo Hwan Kim

Department of Mathematics

Dongeui University

PUSAN 614-714, Korea

E-mail address: sootopology@hanmail.net

YANGKOK KIM

Department of Mathematics

DONGEui University

Pusan 614-714, Korea

E-mail address: ykkim@dongeui.ac.kr 